\title{
Das Zürcher Ressourcen Modell
}

\author{
Maja Storch · Heidi Möller
}

Online publiziert: 23. April 2018

(C) Springer Fachmedien Wiesbaden GmbH, ein Teil von Springer Nature 2018

Den Schwerpunkt des vorliegenden Heftes bilden Artikel, die das Zürcher Ressourcen Modell (ZRM) zum Thema haben. Das ZRM ist ein SelbstmanagementTraining, mit dessen Entwicklung im Jahr 1990 am pädagogischen Institut der Universität Zürich, am Lehrstuhl für pädagogische Psychologie, begonnen wurde. Das ZRM besteht aus einem wissenschaftlich fundierten theoretischen Rahmen und einem darauf aufbauenden Training. Das Training liegt in manualisierter Form vor. ZRM versteht sich als Psychoedukation und bietet den Teilnehmenden Hilfe zur Selbsthilfe. Den Trainer/innen kommt die Rolle der Prozessbegleitung zu, die Inhalte, an denen sie arbeiten wollen, bestimmen die Teilnehmenden und Coachees selbst. Auf diese Art wird ein Training oder ein Coaching mit dem größten Respekt vor der Individualität des einzelnen Menschen durchgeführt. Im Zuge der Entwicklungsarbeiten $\mathrm{zu}$ diesem Training wurden mehrere neue Methoden entwickelt und auf ihre Wirksamkeit hin überprüft.

In diesem Heft sind einige Themen angesprochen, die das breite Spektrum zeigen, in dem ZRM angewendet werden kann. Eine der interessantesten Neuentwicklungen sind die Motto-Ziele. Dieser neue Zieltyp ist Thema der Artikel von Mühlberger et al. und von Dyllick. Christina Mühlberger, Angela Büche und Eva Jonas beschäftigen sich mit dem Einsatz verschiedener Zieltypen bei verschiedenen Typen von Coachees. Nicht jede Art der Zielformulierung ist auch für jeden Typ erfolgversprechend. Im Coaching werden Ziele oft so konkret wie möglich formuliert (z. B. die sogenannten SMART-Ziele). Bei unsicheren und besorgten Coachees ist die

Dr. M. Storch $(\bowtie)$

Scheuchzerstraße 21, 8006 Zürich, Schweiz

E-Mail: maja.storch@ismz.ch 
Identifikation mit dem Ziel zu gering, als dass es motivierend wirkt und handlungswirksam werden kann. Hier ist die Arbeit mit den Motto-Zielen zu empfehlen, die Bewusstes und Unbewusstes in Einklang bringen und so systematisch Motivation und Zielidentifikation erzeugen.

Thomas Dyllick beschreibt in vier Studien seine Untersuchungen und Ergebnisse zur Wirkung des ZRM-Onlinetools zur Entwicklung von persönlichen Motto-Zielen bei unangenehmen Pflichten. Mithilfe dieses Tools können Personen selbstständig in Einzelarbeit - unabhängig von Trainer oder Coach - ein Basismotto-Ziel entwickeln, welches z.B. im Rahmen eines Coachings weiter bearbeitet werden kann. Bereits diese Vorform des Motto-Ziels ändert das subjektive Erleben der Person, hat Effekte auf die Vitalität, reguliert negative Gefühle herunter und bietet eine Möglichkeit zur anstrengungsfreien Selbstregulation. Das ZRM Onlinetool steht kostenlos auf der werbefreien ZRM Homepage (www.zrm.ch) zur Verfügung.

Eine weitere charakteristische Innovation des ZRM ist die Exploration des Unbewussten mit somatischen Markern. Das Unbewusste verfügt nicht über Sprache, es äußert seine Bewertungen über somato-affektive Signale. Im ZRM wird diese Tatsache systematisch genutzt. Es gibt Menschen, die Mühe in der Wahrnehmung und Übersetzung der Signale des Unbewussten haben. Der Fachbegriff heißt Alexithymie (Gefühlsblindheit). Julia Weber beschreibt in ihrem Beitrag, wie die somatischen Marker mit Methoden des ZRM in Sprache übersetzt werden können.

Aus einer Diagnostik nach der Theorie der Persönlichkeits-System-Interaktionen (PSI) von Kuhl ergeben sich viele Bezüge, die direkt in ein ZRM-Training oder Coaching münden können. Corinne Morgenegg gibt in ihrem Artikel eine Einführung in die PSI-Theorie und zeigt auf, wie anhand der diagnostischen Einsichten das ZRM gezielt in eine Standortbestimmung oder eine Laufbahnberatung münden kann.

Change Prozesse sind oft von starken negativen Affekten begleitet, die gemeinhin als „Widerstand“ bezeichnet werden. Wie geht man als Change-Manager/in damit um? Johannes Storch stellt ein Verfahren vor, wie im ZRM konstruktiv und ressourcenaktivierend mit starken negativen Affekten gearbeitet werden kann. Aufbauend auf einem persönlichen Affektziel können Mitarbeitende ethisch respektvoll dabei unterstützt werden, ihre persönliche Haltung zum bevorstehenden Change zu entwickeln, mit der sie gesund und proaktiv arbeiten können.

In den freien Beiträgen befassen sich Sebastian Stelzner und Thomas Bachmann mit der zentralen Frage, was treibt Menschen an, die eine der aktuell angebotenen 260 Coachingweiterbildungen besuchen? In ihrem Beitrag gehen sie dem Motivationsbündel (Bildungs- und Lernmotivation, Steigerung der sozialen Wertigkeit etc.) nach und untersuchen die persönliche, instrumentelle, ökonomische und reflexive Motivation der Weiterbildungsteilnehmer an ihrem Institut. Trotz des Zwangs zur Weiterbildung im Zuge der Subjektivierung von Arbeit, der Notwendigkeit, die eigene Employability sicherzustellen, können die Autoren einen direkten Zusammenhang der Entscheidung für eine Coachingausbildung mit der aktuellen und zukünftigen (beruflichen) Tätigkeit der Teilnehmer/innen feststellen. Das verweist zumindest für das Institut Artop darauf, dass das Scharlatanerieproblem in der Coachingszene nicht zu befürchten ist. 
Der erste Praxisbericht greift das brisante Thema der interkulturellen Dimension in der Beratung erneut auf, das Schwerpunkt des Heftes 1/2018 der OSC war. Emily Slate führt mit ihrem Beitrag das Thema weiter, zeigt uns den Team Power Profiler - ein Internetbefragungstool für virtuelle internationale Teams, das Beratern helfen kann, die Stärken und Schwächen von Teams zu analysieren und diagnosegestützt mit ihnen zu arbeiten. Anhand neun kritischer Erfolgsbereiche, mit denen die meisten Hindernisse zur gelungenen Kooperation beschreibbar sind, lässt sich ein die Beratung strukturierendes Vorgehen planen.

In zweiten Praxisbericht zum Karrierecoaching zur Unterstützung dualer Karrieren im Spitzensport zeigt Moritz Geisreiter auf, welche Rolle der Karriereberatung von gefeierten Spitzensportlern zum Ende ihrer Ruhmesperiode zukommen kann. Wie kann es den Sportlern gelingen, ihre herausragenden Kompetenzen erfolgreich in der Arbeitswelt einzubringen?

Ernst-Dieter Lantermann nimmt in seinem Diskurs Bollwerk Zivilgesellschaft? Der Drang zum Extremen und Fanatismus in unsicheren Zeiten eine sozialpsychologische Analyse unserer Zeit vor. Seine zentrale These ist das Erleben eines fundamentalen Angriffs auf das Selbstwertgefühl vieler Bürger. Lantermann erweitert die gesellschaftliche Bedeutung von Radikalisierungsprozessen im politischen Raum auf den im Streit um die richtige Ernährung und den Tierschutz als weitere extreme Überzeugungssysteme.

In der Rubrik Filmanalysen lassen uns Erhard Tietel und Kornelia Kieper in die spezielle Welt der Gewerkschaften schauen. Brillant zeichnen die beiden Autoren die Geschichte der Gewerkschaften nach, erläutern deren verändertes Selbstverständnis und ihre strukturellen Dynamiken. Sie bieten damit eine gute Orientierung für Beratung und Stakeholder, da die Arbeitnehmervertreter zentrale Player bei allen Innovations- und Changeprojekten sind und in ihre Identität zwischen Co-Management und Interessenvertretung ausloten. Die historischen Anfänge der noch lange nicht beendeten Debatte um Geschlechtergerechtigkeit in Unternehmen werden am Beispiel des „Equal Pay“ mit dem Film über den Streik der Näherinnen in England 1969 nachgezeichnet. 